\title{
Carrier tracking algorithm based on joint acquisition of frequency locked loop and phase locked loop
}

\author{
Kang $\mathrm{Gao}^{1, *}$, Qiang Zhao ${ }^{1}$, BaoTong Peng ${ }^{1}$ \\ ${ }^{1}$ Department of Optical and Electronic Equipment, Aerospace Engineering University, Beijing, 101416, PR China
}

\begin{abstract}
Aiming at the problem of frequency step in the frequency lock loop (FLL) - phase lock loop (PLL) carrier tracking algorithm's conversion state, presenting an improved algorithm: PLL and FLL joint acquisition to replace the single FLL acquires frequency, and deduce the loop state transition threshold. The simulation results show that the improved algorithm is more stable in the conversion process, and the loop performance is optimized. When the SNR is $-10 \mathrm{~dB}$, and has the acceleration rate, the tracking loop does not have a frequency step at the time of conversion, achieving the design purpose.
\end{abstract}

\section{Introduction}

In the space measurement and control system, due to the long distance transmission loss and the high-speed movement of the target carrier, the received signal has the characteristics of low SNR and high dynamic. At present, the receivers in the measurement and control system are mostly coherent receivers, the principle of which is to obtain the coherent carrier with the same frequency and phase to the received signal through the carrier tracking. In the common carrier tracking algorithms, FLL-PLL carrier tracking algorithm has the advantages, such as simple implementation, good dynamic performance and high tracking accuracy. FLLPLL carrier tracking algorithm is one of the tracking algorithms which are widely used.

However, the traditional carrier tracking algorithm of FLL-PLL has the problem of frequency step when converting FLL to PLL. In [1] and [2] which used to adjust the conversion threshold to solve that problem, although this method can improved the frequency step problem, but the effect is not ideal.

This paper aims at the problem of discontinuous transition state, proposed the improved algorithm: FPLLPLL carrier tracking algorithm, the algorithm uses FLL and PLL joint acquisition during the acquisition stage, when converting smoothly into PLL tracking stage. At the same time, the conversion threshold of the algorithm is analysed, which provides a theoretical basis for the design of conversion threshold.

\section{FLL-PLL carrier tracking algorithm}

Figure 1 shows the principle of FLL-PLL tracking loop, which consisted of the discriminator, loop filter (LF) and numerical control oscillator (NCO). From the Figure 1 when the input signal enter the loop, firstly, input signal mixes with the local signal which generated by the NCO, then integral cleaning of the mixed signal, the discriminator can get the phase error or the frequency error from mixed signal, finally, putting the phase error or the frequency error into the loop filter can obtained the NCO frequency control, which makes the output signal of NCO has the same frequency and phase with the received signal, completing carrier tracking.

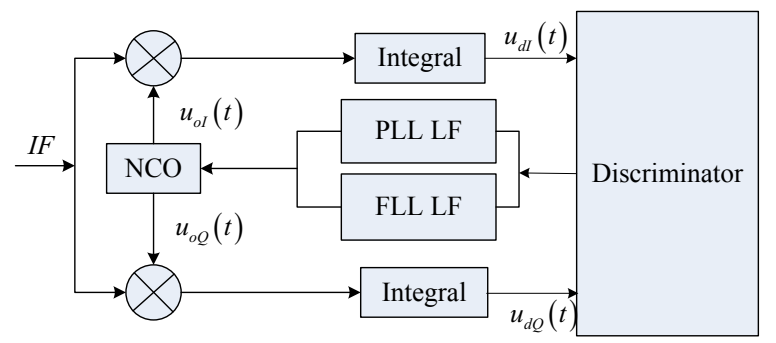

Figure 1. FLL-PLL tracking loop schematic.

\subsection{The design of the discriminator}

FLL-PLL tracking loop discriminator including frequency discriminator and phase discriminator, there are several identification algorithms compared in table 1 and table 2. From table 1 and table 2 can get the form of loop discriminator[3].

Table 1. Commonly used phase recognition algorithm.

\begin{tabular}{|c|c|l|}
\hline Phase algorithm & Output & \multicolumn{1}{|c|}{ Character } \\
\hline$u_{d Q} \times u_{d I}$ & $\sin \left(2 \theta_{e}\right)$ & $\begin{array}{l}\text { In the low SNR } \\
\text { close to the best, } \\
\text { moderate amount } \\
\text { of calculation. }\end{array}$ \\
\hline$u_{d Q} \times \operatorname{sign}\left(u_{d I}\right)$ & $\sin \left(\theta_{e}\right)$ & $\begin{array}{l}\text { In the high SNR } \\
\text { close to the best, } \\
\text { low computational } \\
\text { requirements. }\end{array}$ \\
\hline$u_{d Q} / u_{d I}$ & $\tan \left(\theta_{e}\right)$ & $\begin{array}{l}\text { In the low SNR is } \\
\text { the best, and high } \\
\text { SNR is good, high } \\
\text { computational } \\
\text { requirements. }\end{array}$ \\
\hline $\operatorname{ATAN}\left(u_{d Q} / u_{d I}\right)$ & $\theta_{e}$ & $\begin{array}{l}\text { Two quadrant arc } \\
\text { tangent, in the } \\
\text { high and low SNR } \\
\text { are the best, high } \\
\text { computational } \\
\text { requirements. }\end{array}$ \\
\hline
\end{tabular}

Table 2. Commonly used frequency recognition algorithm

\footnotetext{
* Corresponding author: wouwouwou@126.com
} 


\begin{tabular}{|c|c|l|}
\hline $\begin{array}{l}\text { frequency } \\
\text { algorithm }\end{array}$ & Output & \multicolumn{1}{|c|}{ Character } \\
\hline$\frac{P_{\text {cross }}}{t_{2}-t_{1}}$ & $\frac{\sin \left[\left(\theta_{2}-\theta_{1}\right)\right]}{t_{2}-t_{1}}$ & $\begin{array}{l}\text { In the low } \\
\text { SNR close to } \\
\text { the best, } \\
\text { moderate of } \\
\text { amount of } \\
\text { calculation. }\end{array}$ \\
\hline$\frac{P_{\text {cross }} \times P_{\text {dot }}}{t_{2}-t_{1}}$ & $\frac{\sin \left[2\left(\theta_{2}-\theta_{1}\right)\right]}{t_{2}-t_{1}}$ & $\begin{array}{l}\text { In the high } \\
\text { SNR close to } \\
\text { the best, low } \\
\text { computational } \\
\text { requirements. }\end{array}$ \\
\hline $\begin{array}{l}A T A N 2 \\
\left(P_{\text {cross }}, P_{\text {dot }}\right)\end{array}$ & $\frac{\theta_{2}-\theta_{1}}{t_{2}-t_{1}}$ & $\begin{array}{l}\text { Four quadrant } \\
\text { arc tangent, in } \\
\text { the high and } \\
\text { low SNR are } \\
\text { the best, high } \\
\text { computational } \\
\text { requirements. }\end{array}$ \\
\hline
\end{tabular}

As can be seen from table 1 and table 2 that the discrimination performance of the tangent detection algorithm and the cross product frequency discrimination algorithm are optimal. The structure diagram of discriminator is shown in Figure 2.

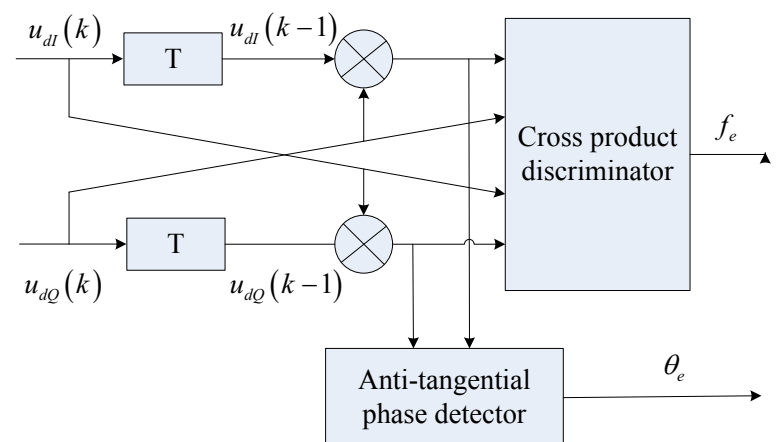

Fig. 1. Discriminator structure.

The received signal is assumed to be of the form

$$
u_{i}(t)=A \cos \left(\omega_{0} t+\varphi_{0}\right)
$$

The NCO output is assumed to be of the form

$$
\begin{aligned}
& u_{o I}(t)=\cos \left(\omega_{1} t+\varphi_{1}\right) \\
& u_{o Q}(t)=\sin \left(\omega_{1} t+\varphi_{1}\right)
\end{aligned}
$$

After the mixing and integral cleaner[4]

$$
\begin{aligned}
& u_{d I}(t)=\frac{1}{T_{S}} \int_{(k-1) N T_{S}}^{k N T_{S}} \frac{A}{2} \cos (\Delta \omega t+\Delta \varphi) d t \\
& u_{d Q}(t)=\frac{1}{T_{S}} \int_{(k-1) N T_{S}}^{k N T_{S}} \frac{A}{2} \sin (\Delta \omega t+\Delta \varphi) d t
\end{aligned}
$$

where: $t_{k}=k N T_{S}, t_{k-1}=(k-1) N T_{S}, T=t_{k}-t_{k-1}$

$$
\begin{aligned}
u_{d I}(t) & =\frac{1}{T_{S}} \int_{(k-1) N T_{S}}^{k N T_{S}} \frac{A}{2} \cos (\Delta \omega t+\Delta \varphi) d t \\
& =\left.\frac{A}{2 T_{S} \Delta \omega} \sin (\Delta \omega t+\Delta \varphi)\right|_{(k-1) N T_{S}} ^{k N T_{S}} \\
& =\frac{A N}{2} \operatorname{sinc} \frac{\Delta \omega T}{2} \cos \left(\Delta \omega t_{k-1}+\frac{\Delta \omega T}{2}+\Delta \varphi\right)
\end{aligned}
$$

and

$$
u_{d Q}(t)=\frac{A N}{2} \operatorname{sinc} \frac{\Delta \omega T}{2} \sin \left(\Delta \omega t_{k-1}+\frac{\Delta \omega T}{2}+\Delta \varphi\right)
$$

$P_{\text {cross }}$ and $P_{d o t}$ are given blow

$$
\begin{aligned}
P_{\text {cross }} & =u_{d I}\left(t_{k-1}\right) u_{d Q}\left(t_{k}\right)-u_{d I}\left(t_{k}\right) u_{d Q}\left(t_{k-1}\right) \\
& =\frac{(A N)^{2}}{4} \operatorname{sinc}^{2} \frac{\Delta \omega T}{2} \sin \Delta \omega T \\
P_{d o t} & =u_{d I}\left(t_{k-1}\right) u_{d I}\left(t_{k}\right)+u_{d Q}\left(t_{k}\right) u_{d Q}\left(t_{k-1}\right) \\
& =\frac{(A N)^{2}}{4} \operatorname{sinc}^{2} \frac{\Delta \omega T}{2} \cos \Delta \omega T
\end{aligned}
$$

The two quadrant tangent phase detection algorithm is used to obtain the phase discrimination error

$$
\theta_{e}=\arctan \left(P_{\text {cross }} / P_{d o t}\right)=\Delta \omega T
$$

The four quadrant tangent frequency detection algorithm is used to obtain the frequency discrimination error

$$
f_{e}=\frac{\arctan 2\left(P_{c r o s s} / P_{d o t}\right)}{\left(t_{k}-t_{k-1}\right) 2 \pi}=\frac{\theta_{e}}{2 \pi T}=\frac{\Delta \omega}{2 \pi}
$$

The phase discrimination error and the frequency discrimination error are optimal under the condition of high and low SNR, and the slope of the identification curve is independent of the signal amplitude.

\subsection{Design of Loop Filter}

When design a carrier tracking loop, the order of the loop filter determines the order of the carrier tracking loop. According to the high dynamic characteristics of the signal in the application background, the FLL-PLL tracking loop needs to overcome the influence of the acceleration and jerk. So it is necessary to design the order of the loop.

Take the second order FLL as an example. Since the FLL tracking loop contains an integrator more than the same order PLL, therefore the error transfer function of the second order FLL with active proportional integral filter is

$$
H_{e}(s)=\frac{K s^{3}}{K s^{3}+2 \xi \omega_{n} s+\omega_{n}^{2}}
$$

where: $K$ is loop gain, $\omega_{n}$ is angular frequency of natural oscillation, $\xi$ is damping coefficient

Under the actual conditions, the mathematical model of the acceleration is the frequency ramp signal, the mathematical model of the jerk is the frequency acceleration signal, the steady-state error of the second order FLL is calculated according to the terminal value theorem in the case of frequency ramp and frequency acceleration:

When the input signal is a frequency ramp signal

$$
\begin{aligned}
f_{e}(\infty) & =\lim _{t \rightarrow \infty} f_{e}(t)=\lim _{s \rightarrow 0} s \bullet f_{e}(s) \\
& =\lim _{s \rightarrow 0} s \cdot \frac{K s^{3}}{K s^{3}+2 \xi \omega_{n} s+\omega_{n}^{2}} \bullet \frac{\dot{f_{d}}}{s^{3}} \\
& =0
\end{aligned}
$$

When the input signal is a frequency acceleration signal 


$$
\begin{aligned}
f_{e}(\infty) & =\lim _{t \rightarrow \infty} f_{e}(t)=\lim _{s \rightarrow 0} s \bullet f_{e}(s) \\
& =\lim _{s \rightarrow 0} s \cdot \frac{K s^{3}}{K s^{3}+2 \xi \omega_{n} s+\omega_{n}^{2}} \bullet \frac{\ddot{f_{d}}}{s^{4}} \\
& =\frac{K \dot{f_{d}}}{\omega_{n}^{2}}
\end{aligned}
$$

From (13) and (14), we can see that the second order FLL can track the frequency ramp signal without error, but the steady frequency error occurs when tracking the frequency acceleration signal.

Take the third order PLL as an example. The error transfer function of the third order PLL with three parameter filter[5] is

$$
H_{e}(s)=\frac{K s^{3}}{K s^{3}+2 \xi \omega_{n} s+\omega_{n}^{2}}
$$

where:

$K$ is loop gain,

$$
\begin{aligned}
& a=(m+2) \xi \omega_{n} / K, \\
& b=(1+2 m \xi) \omega_{n}^{2} / K, \\
& c=m \xi \omega_{n}^{3} / K
\end{aligned}
$$

When the input signal is a frequency ramp signal

$$
\begin{aligned}
\theta_{e}(\infty) & =\lim _{t \rightarrow \infty} \theta_{e}(t)=\lim _{s \rightarrow 0} s \bullet \theta_{e}(s) \\
& =\lim _{s \rightarrow 0} s \cdot H_{e}(s) \cdot \frac{\dot{f}_{d}}{s^{3}} \\
& =\lim _{s \rightarrow 0} s \cdot \frac{s^{3}}{s^{3}+s^{2} K a+s K b+K c} \cdot \frac{\dot{f}_{d}}{s^{3}} \\
& =0
\end{aligned}
$$

When the input signal is a frequency acceleration signal

$$
\begin{aligned}
\theta_{e}(\infty)= & \lim _{t \rightarrow \infty} \theta_{e}(t)=\lim _{s \rightarrow 0} s \cdot \theta_{e}(s) \\
= & \lim _{s \rightarrow 0} s \cdot H_{e}(s) \cdot \frac{\ddot{f_{d}}}{s^{4}} \\
= & \lim _{s \rightarrow 0} s \cdot \frac{s^{3}}{s^{3}+s^{2} K a+s K b+K c} \cdot \frac{\ddot{f_{d}}}{s^{4}} \\
& \stackrel{f_{d}}{K c}
\end{aligned}
$$

The computed results show that in the traditional FLLPLL carrier tracking algorithm, firstly, the FLL to capture, from (14) we can see that the second order FLL will have a stable frequency error, at this time FLL compensates for acceleration and jerk, for the third-order PLL there is only a frequency step to track, so in the third-order PLL separate work stage, PLL can be accurate tracking without error. Therefore, FLL and PLL loop filter design process, as discussed above, can determine the second-order FLL and third-order PLL combination of the form.

\section{FPLL-PLL carrier tracking algorithm}

On the basis of guaranteeing the locking speed and tracking accuracy, the FPLL-PLL carrier tracking algorithm can make the conversion state more continuous and the loop is more stable. In the FLL-PLL carrier tracking algorithm, FLL captures Doppler frequency at first, when being stable to only a steadystate frequency error, then the loop is converted to PLL for accurate tracking. Because of the conversion mode for the forced convert, it is clear that the transition of the loop state will not continuous, thus affecting the tracking performance of the loop, or even cause the phenomenon of loss of lock.

The FPLL-PLL carrier tracking algorithm uses the FLL and PLL joint acquisition. At this stage, the PLL assists FLL to acquire Doppler frequency, in this way can avoid the problem of frequency step due to forced conversion and then the loop converts to eh exact tracking mode for the PLL, until the loop error is too large, resulting in loss of lock.

\subsection{Dynamic performance of FPLL}

In order to ensure the locking speed of FPLL-PLL carrier tracking algorithm, it is necessary to analyse the jerk stress threshold that FPLL and compare with FLL. The jerk stress threshold under different carrier-to-noise ratios can be deduced from the total tracking loop measurement error. The jerk stress threshold of secondorder FLL and third-order PLL are given blow.

The dominant source of the FLL frequency error are frequency jitter due to thermal noise and dynamic stress errors. The rule of thumb for tracking threshold is not exceed one-fourth of the frequency pull-in range of the FLL discriminator[6]. Therefore, the general rule of thumb for FLL tracking threshold is:

$$
\sigma_{F L L}=\sigma_{t F L L}+f_{e} / 3 \leq 1 / 12 T
$$

where:

$$
\begin{aligned}
& \sigma_{t F L L}=1 \text {-sigma thermal noise frequency jitter } \\
& f_{e}=\text { dynamic stress error in the FLL tracking loop }
\end{aligned}
$$

The FLL tracking loop jitter due to thermal noise is:

$$
\sigma_{t F L L}=\frac{1}{2 \pi T} \sqrt{\frac{4 F B_{L}}{C / N_{0}}\left[1+\frac{1}{T C / N_{0}}\right]}
$$

where:

$$
F=1 \text { at high } C / N_{0}
$$

$=2$ near threshold

The dynamic stress error is:

$$
f_{e}=0.2809 \frac{\dot{f_{d}}}{B_{L}^{2}}
$$

From (18), (19), (20) the jerk stress threshold of FLL is defined as follows:

$$
\ddot{f_{d}} \leq \frac{3}{0.2809}\left(\frac{B_{L}^{2}}{12 T}-\frac{B_{L}^{2}}{2 \pi T} \sqrt{\frac{4 F B_{L}}{C / N_{0}} \frac{T C / N_{0}+1}{T C / N_{0}}}\right)
$$

In the case of a dataless PLL four-quadrant arctangent discriminator whose phase pull-in range is $360^{\circ}$, the 3 sigma rule threshold is therefore $90^{\circ}$ [6]. Therefore, the general rule of thumb for FLL tracking threshold is: 


$$
\sigma_{P L L}=\sigma_{t P L L}+\theta_{e} / 3 \leq 30^{\circ}
$$

where:

$\sigma_{t P L L}=1$-sigma thermal noise phase jitter

$\theta_{e}=$ dynamic stress error in the PLL tracking loop

The FLL tracking loop jitter due to thermal noise is:

$$
\sigma_{t P L L}=\frac{360}{2 \pi} \sqrt{\frac{B_{L}}{C / N_{0}}\left[1+\frac{1}{2 T C / N_{0}}\right]}
$$

The dynamic stress error is:

$$
\theta_{e}=0.4828 \frac{\dot{f_{d}}}{B_{L}^{3}}
$$

From (21), (22), (23) the jerk stress threshold of PLL is defined as follows:

$$
\ddot{f_{d}} \leq \frac{3}{0.4828}\left(30 B_{L}^{3}-\frac{360 B_{L}^{3}}{2 \pi} \sqrt{\frac{B_{L}}{C / N_{0}}\left[1+\frac{1}{2 T C / N_{0}}\right]}\right)
$$

According to the Monte Carlo simulation experiment and the experience value of the engineering experiment, we can get the relationship between the jerk stress threshold and the loop bandwidth of FLL and FPLL under $\mathrm{SNR}=-10 \mathrm{~dB}[7]$, as shown in the following figure

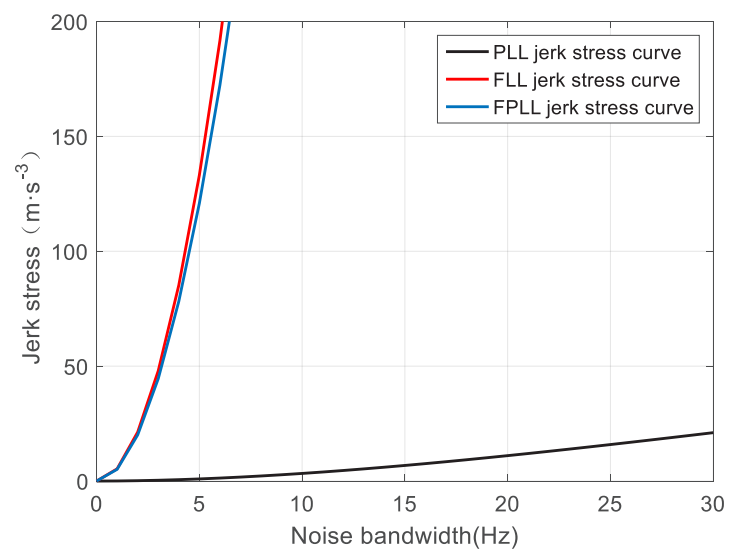

Fig 3. Jerk stress threshold of FLL and FPLL.

In summary, compared the jerk stress threshold of FPLL and FLL, FPLL's jerk stress threshold meets the actual conditions and enable guarantee loop locking.

\subsection{Transition threshold}

Whatever the traditional FLL-PLL carrier tracking algorithm or the FPLL-PLL carrier tracking algorithm, the choice of conversion timing is a key factor affecting loop tracking performance. Premature or late switch to the PLL accurate tracking state will affect the performance of the loop, and even cause the loop to lose the lock.

The threshold for converting the FPLL state to the PLL exact tracking state can be set to fast capture of the PLL. Because in the fast catch band, the PLL can't be jumped to reach the lock.

The high frequency component of the three-parameter filter transfer function can be shown to be

$$
\lim _{s \rightarrow \infty} F(s)=\lim _{s \rightarrow \infty} \frac{a s^{2}+b s+c}{s^{2}}=a
$$

where: $m=5$, the gain of the high frequency part of the loop is can be shown to be

$$
K_{H}=K a=(m+2) \zeta \omega_{n}
$$

(27) is the maximum direct NCO frequency control range that is fast capture, can be shown to be

$$
\Delta \omega_{L}=K a=(m+2) \zeta \omega_{n}
$$

In summary, when the FPLL's steady-state error reaches the threshold, the FLL in the FPLL stop working, the loop is converted to PLL accurate tracking stage.

\section{Simulation and analysis}

The analytical simulation results for verification. For example, a sinusoidal signal with a frequency of $70 \mathrm{MHz}$, and the sampling rate is $40 \mathrm{MHz}$, the initial Doppler frequency difference is $200 \mathrm{~Hz}$, the Doppler first order change rate is $50 \mathrm{~Hz} / \mathrm{s}$, the Doppler second order change rate is $5 \mathrm{~Hz} / \mathrm{s}^{2}$, under the condition of SNR is $-10 \mathrm{~dB}$, using FLL-PLL and FPLL-PLL tracking the above signal. The simulation results are shown in the following figure

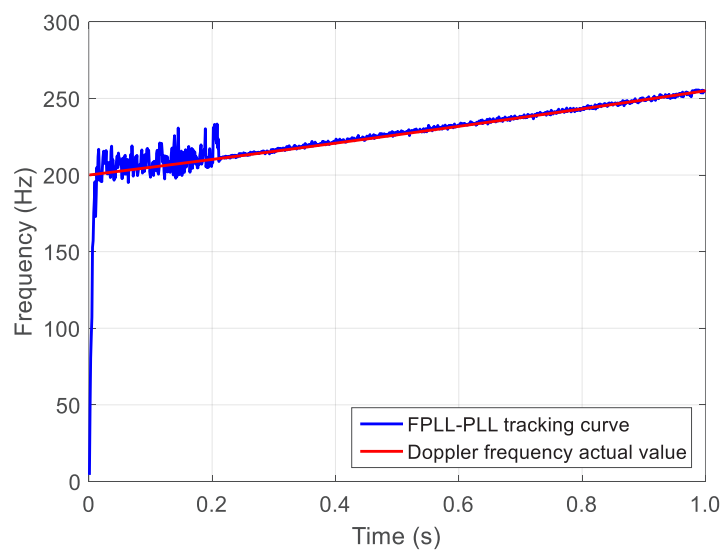

Fig 4. Output of FPLL-PLL loop filter.

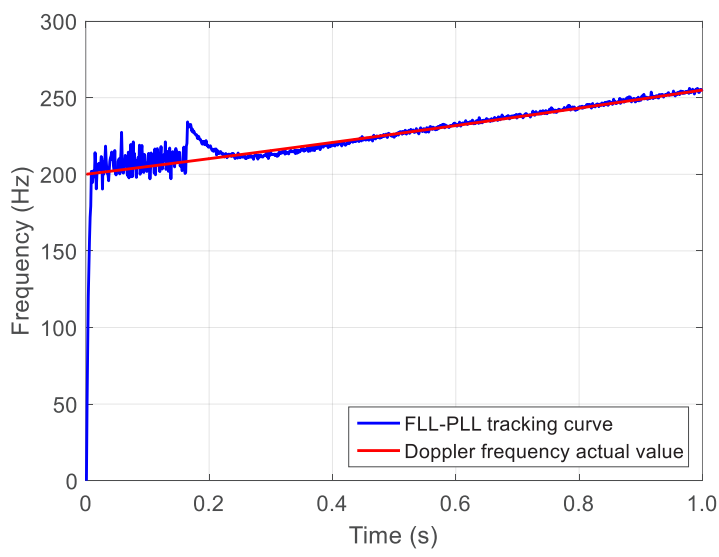

Fig 5. Output of FLL-PLL loop filter.

It can be seen from Figure 4 and Figure 5 that the conversion state of the FPLL-PLL carrier tracking algorithm is more continuous and the loop is more stable 
under the low SNR. The traditional FLL-PLL carrier tracking algorithm takes a frequency step in the conversion state, the loop stability is relatively poor.

\section{Conclusions}

The FPLL-PLL carrier tracking algorithm proposed in this paper uses the method of PLL and FLL joint acquisition, improving the loop conversion state, compared with the traditional FLL-PLL carrier tracking algorithm, the loop conversion is more continuous and the loop stability is higher.

However, this paper only has improved the frequency step problem of carrier tracking loop conversion, and it has not been studied for the variable bandwidth problem in the precise tracking stage. This will be the next step to be improved.

\section{References}

1. Z. P. Xu, C. Cui, J . She, Telecommunication Engineering, 52, (2012), 558-561.

2. H. M. Wu, Y. Yong. Su, A. J. Liu, Science Technology and Engineering, 10, (2010), 4645-4650

3. H. B. Lu, W. W. Wang, Q. C. Gu, Information and Control, 39, (2010), 451-454.

4. F. D. Natali, AFC tracking algorithms[J]. IEEE Transactions on Communications, 32, (1984), 935947.

5. Y. Z. Wang, Telecommunication Engineering, 48, (2008), 51-55.

6. E. D. Kaplan, Journal of Atmospheric and SolarTerrestrial Physics, 59, (2006):598-599.

7. X. D. Deng, S. Wu, Modern Defence Technology, 38, (2010), 137-141. 